\title{
Cordotomy for treatment of cancer-related pain: patient selection and intervention timing
}

\author{
Ashwin Viswanathan, M.D., ${ }^{1}$ and Eduardo Bruera, M.D. ${ }^{2}$ \\ Departments of ${ }^{I}$ Neurosurgery and ${ }^{2}$ Palliative Care \& Rehabilitation Medicine, The University of Texas MD \\ Anderson Cancer Center, Houston, Texas
}

\begin{abstract}
Many neurosurgical interventions for the management of cancer-related pain have been tried, but their role in today's advanced supportive and palliative care is not well described. The authors discuss the current knowledge gaps that prevent successful integration of neurosurgical interventions and patients with cancer-related pain.

Two patients underwent percutaneous CT-guided cordotomy for refractory cancer-related pain: one patient had melanoma and the other had ovarian carcinoma. Both patients seemed to have unilateral, somatic, nociceptive cancerrelated pain.

Cordotomy was effective for only 1 patient.

Percutaneous CT-guided cordotomy is a low-risk intervention that can benefit carefully selected patients with cancer-related pain. There is a clear need for prospective controlled studies to evaluate the effectiveness of cordotomy for patients receiving optimal medical treatment. A multidisciplinary study design could help to identify factors correlated with a positive outcome.

(http://thejns.org/doi/abs/10.3171/2013.6.FOCUS13237)
\end{abstract}

KEY WORDS • pain • cordotomy • cancer-related pain

$\mathrm{W}$ ORLD Health Organization guidelines initially published in 1986 serve as a foundation for the treatment of cancer-related pain..$^{10}$ When these recommendations are followed, pain relief is achieved for an estimated $70 \%-90 \%$ of cancer patients. However, controlled studies of pain relief achieved by using the WHO recommendations have been difficult to perform. ${ }^{1,4}$ Data suggest that medical undertreatment of pain may continue to be a factor in inadequate cancer pain management. ${ }^{2}$ Even among optimally treated patients, some continue to have high scores for pain or opioid-induced toxicity.

For the management of certain cancer pain syndromes, several targeted procedural interventions have been proposed. Few high-quality studies have assessed the effectiveness of these interventions, and information to help with patient selection or determination of optimal timing of the procedure is limited. One procedural intervention, for which significant Class III evidence has been published, is percutaneous cordotomy. ${ }^{9}$

Cordotomy involves creating a lesion of the lateral spinothalamic tract, which is located in the anterolateral quadrant of the spinal cord. It is estimated that $20 \%$ of

Abbreviation used in this paper: $\mathrm{MEDD}=$ morphine equivalent daily dose. the spinal cord must be ablated to achieve adequate pain relief with cordotomy. ${ }^{7}$ Several techniques exist for performing cordotomy, including open surgical exposure and mechanical lesioning of the spinothalamic tract, and percutaneous radiofrequency techniques with use of fluoroscopy or CT. Percutaneous CT-guided cordotomy at $\mathrm{C} 1-2$ is currently the standard technique used. The goal of cordotomy is to achieve diminished pinprick sensation in the painful region of the body.

Early reports indicated that the complication rate for open and percutaneous cordotomy was significant: the incidence of short-term limb weakness was high (up to $70 \%$ ). However, in the era of CT-guided techniques, this complication rate has become very low $(<1 \%)$. Current evidence suggests that CT-guided cordotomy might be an effective intervention for patients with cancer-related pain. ${ }^{5,8}$ We present 2 cases that highlight the continuing challenges of patient selection and timing of the cordotomy procedure.

\section{Case Reports}

\section{Case 1}

A 35-year-old man with metastatic melanoma was 
hospitalized because of pain in the right leg. Melanoma of the right lower leg had been diagnosed 7 years earlier, and the patient underwent multimodal therapy, including surgery, radiation therapy, and chemotherapy. Beginning 3 months before this hospitalization, increasing pain developed in the right lower extremity, associated with significant soft-tissue tumor involvement (Fig. 1). His pain was especially difficult to control during wound dressing changes. During this hospitalization, the patient's pain was not controllable with opioids, including $5000 \mu \mathrm{g}$ per day of intravenous fentanyl in addition to $480 \mathrm{mg}$ per day MEDD for other opioids. The neurosurgical service was consulted, and the patient underwent a percutaneous cordotomy (Fig. 2) without complication; 3 days later, he was discharged to home hospice care. Pain control was excellent, and pain intensity score during dressing changes went from a 10 of 10 preoperatively to 2 of 10 postcordotomy. In addition, his MEDD was reduced by $75 \%$.

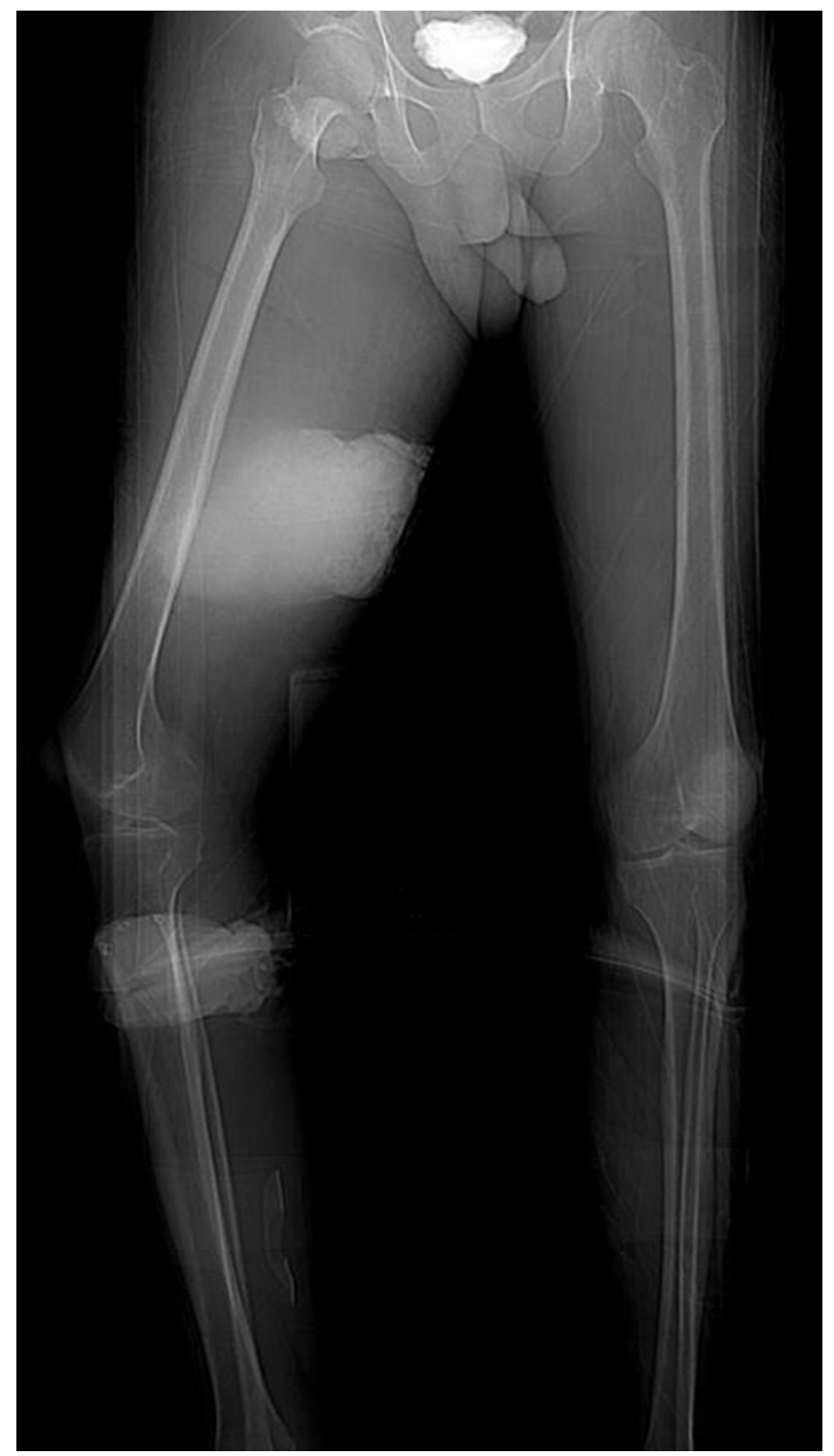

FIG. 1. Case 1. Scout view CT image of the lower extremities, demonstrating extensive soft-tissue disease in the right lower extremity.

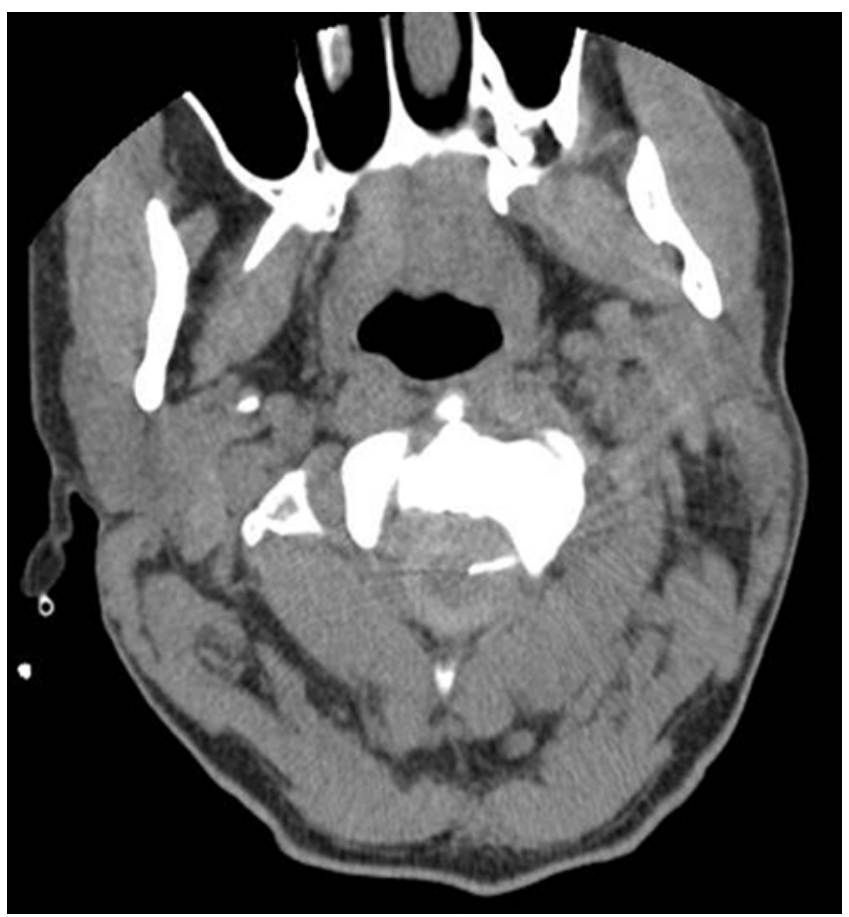

FIG. 2. Case 2. Axial CT image at the level of $\mathrm{C} 1-2$, demonstrating the radiofrequency cordotomy electrode positioned within the left lateral spinothalamic tract.

However, because of disease progression, the patient died on postcordotomy Day 10.

\section{Case 2}

A 48-year-old woman with progressive stage IIIC ovarian carcinoma was hospitalized because of intractable left abdominal and leg pain. The cancer had been diagnosed 4 years earlier, and the patient had undergone surgery, radiation therapy, and chemotherapy. Her current pain was attributed to a mass in her left psoas muscle. As a supportive care service patient, she received multimodal supportive care, including management with opioids. Despite optimal medical management, which included hydromorphone with an MEDD of $300 \mathrm{mg}$, she reported a pain intensity of $9 / 10$. At the request of the medical oncologist, the patient underwent a percutaneous cordotomy. After the procedure, the patient did not experience significant pain relief and she was transitioned to hospice care. Her hydromorphone was changed to the subcutaneous route at the same MEDD. She died at home 1 month later.

\section{Discussion}

In most centers, opioids are, appropriately, the main treatment for cancer-related pain. The WHO recommendations are centered on 5 phrases: "by the mouth," "by the clock," "by the ladder," "for the individual," and "attention to detail," which are guiding principles for the management of opioids for patients with cancer. ${ }^{10}$ Opioids used to treat cancer pain are typically pure $\mu$-agonists, and individualizing therapy is important because of dif- 


\section{Cordotomy: patient selection and intervention timing}

ferences in how a given patient responds to the various opioids. ${ }^{3,6}$ However, when a patient is intolerant of opioids or does not achieve pain relief with maximal supportive care measures, interventional procedures should be considered.

Case 1 serves as an excellent example of a patient who might benefit from a percutaneous cordotomy. The patient had unilateral, cancer-related nociceptive pain. His profound pain relief after cordotomy indicates that this intervention could have been considered earlier, which might have improved his quality of life and decreased health care costs (fewer emergency room visits and hospitalizations for pain control).

In contrast, the patient in Case 2 did not experience any clinically significant benefit from percutaneous cordotomy. Before the intervention, the patient had been evaluated according to the Edmonton Symptom Assessment Scale and was found to have depression rated at 6 of 10 and anxiety rated at 5 of 10. Despite extensive supportive counseling and medical management, her psychological symptoms and a likely suffering component to her pain ultimately might have limited any potential benefit of an interventional procedure. This case emphasizes that a multidisciplinary team is essential for identifying patients with barriers to intervention success.

Two large studies of percutaneous cordotomy have been published..$^{5,8}$ In 2009, Kanpolat and colleagues ${ }^{5}$ published their 20-year experience with cordotomy, describing 193 patients who underwent CT-guided percutaneous cordotomy for cancer pain. The most common sources of pain were pulmonary malignancies, mesothelioma, gastrointestinal tumors, and Pancoast tumor. Median visual analog scale scores were 8 preoperatively and 0 postoperatively. Karnofsky Performance Scale scores also improved significantly; median scores were 40 preoperatively and 70 postoperatively. Unfortunately, the authors do not detail the longer-term outcomes. No permanent neurological injuries resulted from the procedure, and incidence of temporary motor dysfunction and ataxia was low (2.4\%). Dysesthetic pains after the procedure were experienced by $2 \%$ of patients.

In 2008, Raslan ${ }^{8}$ reported on 41 patients who had undergone percutaneous cordotomy for the treatment of cancer-related pain; almost half of these patients had chest wall pain resulting from mesothelioma. Postoperatively, $80 \%$ of patients had no pain, and this effect lasted another month. At 6 months after the procedure, 32\% of patients had no pain, and another $48 \%$ had partial satisfactory pain relief. Other improvements included a significant increase in the total number of hours spent sleeping and a significant improvement in Karnofsky Performance Scale scores.

Although these 2 studies provide supportive evidence for the efficacy of cordotomy, the applicability of these data to patients today is unclear. Many cancer patients in Turkey and Egypt do not have access to supportive care services, including optimal opioid therapy. In contrast, the WHO guidelines are well used in the US, and most patients with cancer-related pain, especially those in a specialty cancer hospital, appropriately receive opioids early. A remaining question with regard to evaluating these 2 studies is whether the patients would have done as well with optimal opioid therapy provided by a trained supportive care team, obviating the need for procedural interventions.

Data from a large tertiary cancer care center emphasize that adequate pain management for cancer patients requires time and dedication from the treatment team. ${ }^{11}$ Of 1612 patients consecutively seen at an outpatient supportive care center, $45 \%$ had achieved pain relief by their first follow-up visit. These patients would probably never need a procedural intervention because of their prompt and effective response to opioids. However, at the first follow-up visit, $31 \%$ of patients continued to have pain scores of 4 and above, and $32 \%$ of patients who initially reported mild pain reported worsened pain at their first follow-up visit. These patients might benefit from an early neurosurgical evaluation and possible intervention.

There is a clear need for a prospective controlled study to evaluate the effectiveness of cordotomy in patients receiving optimal medical treatment. Although multidimensional symptom scale scores for the 2 patients reported here differed, from the standpoint of pain etiology it is not clear how Case 1 differed from Case 2. A multidisciplinary effort to evaluate outcomes and identify those patient characteristics that correlate with a positive patient outcome is essential.

\section{Conclusions}

Although WHO treatment guidelines continue to serve as an important foundation for treatment, ongoing efforts to minimize cancer-related pain are essential. Percutaneous CT-guided cordotomy is a low-risk intervention that might help reduce cancer pain in appropriately selected patients. The 2 cases reported here highlight the continuing challenge of patient selection and timing of neurosurgical consultation. Prospective, randomized, controlled trials are essential for answering these questions.

\section{Disclosure}

The authors report no conflict of interest concerning the materials or methods used in this study or the findings specified in this paper.

Author contributions to the study and manuscript preparation include the following. Drafting the article: Viswanathan. Critically revising the article: Bruera.

\section{References}

1. Azevedo São Leão Ferreira K, Kimura M, Jacobsen Teixeira M: The WHO analgesic ladder for cancer pain control, twenty years of use. How much pain relief does one get from using it? Support Care Cancer 14:1086-1093, 2006

2. Deandrea S, Montanari M, Moja L, Apolone G: Prevalence of undertreatment in cancer pain. A review of published literature. Ann Oncol 19:1985-1991, 2008

3. Fine PG, Portenoy RK: Establishing "best practices" for opioid rotation: conclusions of an expert panel. J Pain Symptom Manage 38:418-425, 2009

4. Jadad AR, Browman GP: The WHO analgesic ladder for cancer pain management. Stepping up the quality of its evaluation. JAMA 274:1870-1873, 1995 
5. Kanpolat Y, Ugur HC, Ayten M, Elhan AH: Computed tomography-guided percutaneous cordotomy for intractable pain in malignancy. Neurosurgery 64 (3 Suppl):ons187-ons194, 2009

6. Knotkova H, Fine PG, Portenoy RK: Opioid rotation: the science and the limitations of the equianalgesic dose table. $\mathbf{J}$ Pain Symptom Manage 38:426-439, 2009

7. Lahuerta J, Bowsher D, Lipton S, Buxton PH: Percutaneous cervical cordotomy: a review of 181 operations on 146 patients with a study on the location of "pain fibers" in the C-2 spinal cord segment of 29 cases. J Neurosurg 80:975-985, 1994

8. Raslan AM: Percutaneous computed tomography-guided radiofrequency ablation of upper spinal cord pain pathways for cancer-related pain. Neurosurgery 62 (3 Suppl 1):226-234, 2008

9. Raslan AM, Cetas JS, McCartney S, Burchiel KJ: Destructive procedures for control of cancer pain: the case for cordotomy. A review. J Neurosurg 114:155-170, 2011
10. World Health Organization: Cancer Pain Relief: With a Guide to Opioid Availability, ed 2. Geneva: World Health Organization, 1996

11. Yennurajalingam S, Kang JH, Hui D, Kang DH, Kim SH, Bruera E: Clinical response to an outpatient palliative care consultation in patients with advanced cancer and cancer pain. J Pain Symptom Manage 44:340-350, 2012

Manuscript submitted May 15, 2013.

Accepted June 13, 2013.

Please include this information when citing this paper: DOI: 10.3171/2013.6.FOCUS13237.

Address correspondence to: Ashwin Viswanathan, M.D., MD Anderson Cancer Center, 1515 Holcombe Blvd., Houston, TX 77030. email: aviswanathan@mdanderson.org. 